\title{
Erythropoietin Therapy for Premature Infants: Cost without Benefit?
}

\begin{abstract}
A review of Donato H, Vain N, Rendo P, et al. 2000 Effect of early versus late administration of human recombinant erythropoietin on transfusion requirements in premature infants: results of a randomized, placebo-controlled, multicenter trial. Pediatrics 105: 1066-1072.
\end{abstract}

$\mathrm{P}_{\mathrm{r}}^{\mathrm{r}}$ REMATURE INFANTS, PARTICULARLY those requiring intensive care, frequently develop anemia. This results primarily from blood sampling and also from a relatively poor erythropoietic response to anemia. As a result, these infants often receive multiple transfusions with the risk of disease transmission. It had been hoped that enhancing erythropoiesis by the administration of erythropoietin (Epo) would prevent or reduce the need for blood transfusions.

There have been many studies of Epo therapy in premature infants with varying results. The most recent of these is that reported by Donato et al. (1) in which they describe a multicenter study carried out in seven hospitals in Buenos Aires. The question asked in that study was whether erythropoietin, given early in life (from Day 2 ), would reduce the need for transfusions in the first 2 weeks of life. They included 114 infants of birth weight less than 1250 grams, half of whom received Epo (a relatively high dose of 1250 units/Kg/week, IV) from Day 2 to 14 and half received no Epo during that period. All infants received Epo (750 units/Kg/week, SC) from Day 14 for an additional 6 weeks.

Those infants who received Epo in the first 2 weeks had significantly greater reticulocyte counts and less of a fall in hematocrit levels. However there was no difference in the total number of transfusions given. Those infants whose birth weights were less

\section{Alvin Zipursky}

than 800 grams required significantly more transfusions during their entire hospital stay; the group which received Epo early required $3.4+/-1.1$ transfusions vs those who received Epo after 14 days $(5.4+/-3.7)(\mathrm{p}<.05)$.

These findings are consistent with many previous studies: Epo does not prevent the early $(<2$ weeks) anemia of premature infants, but may reduce the subsequent number of transfusions given. However it should be made clear that the goal of Epo therapy is not a reduction in the number of transfusions but rather a reduction in the number of blood donors to which the infant is exposed. Current techniques for transfusion of premature infants permit the use of one unit of blood for multiple transfusions of premature infants, for as long as 42 days (2). Using this technique of "dedicated donations," only a small minority of premature infants would be exposed to the blood of more than one donor. Accordingly, it can be concluded that Epo is of little, if any, benefit in reducing exposure of premature infants to the risks of multiple transfusions. But can it protect these infants from receiving any transfusions? The vast majority of premature infants who require transfusions receive their initial transfusion in the first 2 weeks of life (I studied a small series in our hospital and found that
91/98 premature infants who were transfused received their first transfusion before Day 14). Since Epo does not affect the number of transfusions during that period, it is unlikely that Epo will protect infants from receiving their first transfusion.

Using modern transfusion therapy, Epo does not protect premature infants from the risks of single or multiple blood transfusions and therefore there is little or no indication for Epo therapy in the treatment of premature infants. Furthermore Epo is expensive and has known and potential side effects. A reduction in donor exposure is best achieved by reducing the amount of blood taken from these infants, and when transfusion is required "dedicated" units should be used so that the infant will be exposed to the blood of only one donor.

\footnotetext{
1. Donato H, Vain N, Rendo P, Vivas N, Prudent L, Larguía M, Digregorio J, Vecchiarelli C, Valverde R, García C, Subotovsky P, Solana C, Gorenstein $\mathrm{A}$, and for the Private Hospitals Neonatal Network 2000 Effect of early versus late administration of human recombinant erythropoietin on transfusion requirements in premature infants: results of a randomized, placebo-controlled, multicenter trial. Pediatrics 105:1066-1072

2. Lee DA, Slagle TA, Jackson TM, Evans CS 1995 Reducing blood donor exposure in low birth weight infants by the use of older, unwashed packed red blood cells. J Pediat 126:280-286
}

The Hospital for Sick Children

555 University Avenue

Toronto, Ontario

Canada M5G 1 X8 